\title{
The Influence of Electrohydrodynamic Secondary Flow on the Collection Efficiency and Deposition Pattern in ESP
}

\author{
Jun Guo, ${ }^{1,2}$ Xinglian Ye ${ }^{D},{ }^{1}$ Shuai Wang, ${ }^{1,2}$ Baoyu Guo, \\ Chucheng Zhang, ${ }^{1,2}$ and Yinbiao $\mathrm{Su}^{1,2}$ \\ ${ }^{1}$ Fujian Longking Co., Ltd., Longyan, Fujian 36400, China \\ ${ }^{2}$ State Environmental Protection Engineering Center for Power Industrial Dust Control, Longyan, Fujian 36400, China \\ ${ }^{3}$ Department of Chemical Engineering, Monash University, Clayton, VIC 3800, Australia \\ Correspondence should be addressed to Xinglian Ye; yexinglian1228@126.com
}

Received 9 May 2019; Revised 21 June 2019; Accepted 27 June 2019; Published 14 July 2019

Academic Editor: Dongmin Yang

Copyright (C) 2019 Jun Guo et al. This is an open access article distributed under the Creative Commons Attribution License, which permits unrestricted use, distribution, and reproduction in any medium, provided the original work is properly cited.

\begin{abstract}
Electrostatic precipitator (ESP) is widely used for dust removal from flue gas in industry. In the electrostatic precipitation, the electrohydrodynamic secondary flow (EHD) produced by corona ionization has an important influence on the characteristics of particle transport and the collection efficiency of ESP. In this work, a comprehensive ESP model with interaction of multiple physical fields is established to study the EHD effect in ESP. The numerical results show that the EHD generally can increase the streamwise velocity of airflow near the collection plate, which makes the removal performance of ESP worsen. Meanwhile, the EHD has a significant effect on the particle deposition pattern, especially at lower flue gas velocity. When the needle tip of discharge electrode points to the collection plate, the EHD can promote the circulation of airflow near the corona wire, increase the probability of particle charging, and then improve the collection efficiency of ESP.
\end{abstract}

\section{Introduction}

Electrostatic precipitator (ESP) is widely used to purify the flue gas in the thermal power plants. In electrostatic precipitation, the force driving gas-solid separation just acts on the solid particles; hence the ESP has the advantages of less energy cost and pressure loss, high collection efficiency, and good applicability for particles with different physical characteristics. Furthermore, high collection efficiency can be achieved under conditions of high temperature and pressure [1-4]. Nevertheless, the low removal efficiency on particles less than $10 \mu \mathrm{m}$ in ESP still exists. And it is necessary to improve the removal performance of ESP for conforming the strict flue gas emission standards.

Massive free ions resulting from the corona discharge move along the electric line and collide with neutral molecules in the air, which transfers the momentum from ions to neutral molecules, known as electrohydrodynamic secondary flow (EHD) [5-9]. The significant effect of EHD on the transport of particles, especially fine particles, has been confirmed in previous studies [10-13]. And the particle transport is closely related to the collection efficiency used for assessing removal performance of ESP. Therefore, the effect of EHD should be considered in optimal design of ESP.

Some scholars characterized the EHD phenomenon of ESP experimentally. Leonard et al. performed the laseranemometry experiments and demonstrated that a 2-D positive corona discharge has an interaction with the flow of a wire-plate ESP by a nonturbulent secondary flow in a plane perpendicular to the discharge wires. A basic conclusion was verified that negative-corona-discharge nonuniformities are responsible for producing both secondary flows in a plane parallel to the discharge wire [14]. Davidson et al. explained the EHD flow by measured current-voltage relationships, turbulence intensities, integral length scales, and eddy diffusivities. And by comparison with gas flow in the wire-plate precipitator, they found that increases in turbulence intensity in the barbed plate geometry are offset by reductions in the scale of the electrically induced flow [15]. Kallio et al. characterized the complex flow field in a simple, three-wire precipitator by flow visualization, electrostatic and fluid dynamic numerical modelling, and 


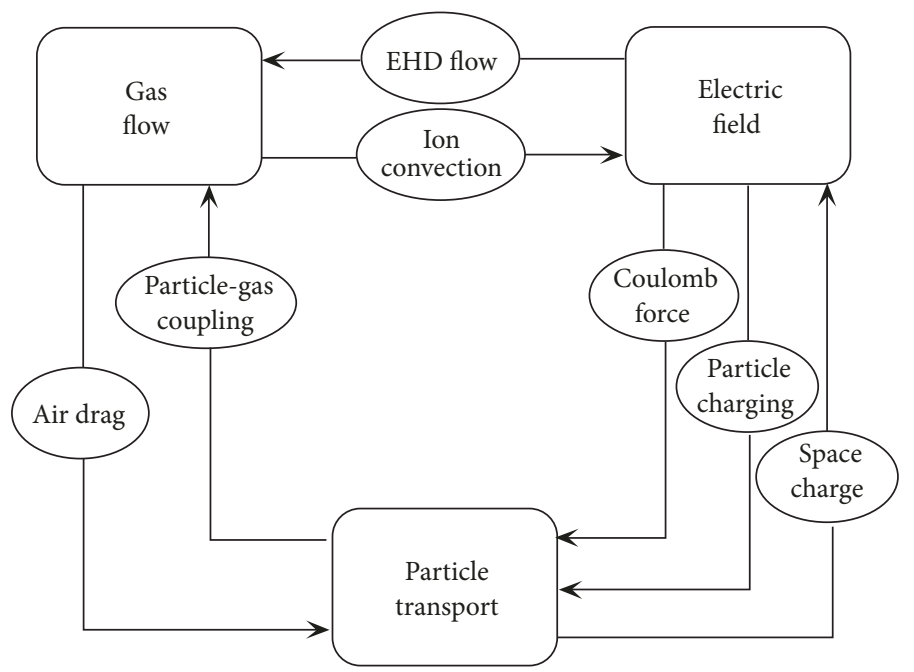

FIGURE 1: Multifield coupling in ESP.

laser-Doppler anemometry (LDA). And the measurement result of streamwise velocity showed that flow acceleration zones occurred upstream of each wire and also between wires near the collecting plate [16].

In experimenting, the accuracy of Laser Doppler Anemometer and hot wire probe can be interfered by the external electric field. Taking this into account, numerical simulation has been favored by more researchers. Bernstein et al. adopted a fundamental model being laid on the coupled effects of the fluid dynamics and electrostatics and found that the interactions between fluid dynamics and electrostatics have a significant role in altering the flow in the precipitator [17]. Zhao et al. employed a hybrid finite-element methodmethod of characteristics numerical algorithm to study the interaction between the electric and the flow fields. And they found that the EHD flow plays an important role in the channel at very low Reynolds number and high EHD characteristic number [18]. Heng et al. studied the EHD in wire-plate ESP of five shaped collecting electrodes with the aid of a user defined function (UDF) and drew the conclusion that the EHD induced in the C-type plate is the strongest among the five channels [8]. Mantach et al. investigated the effect of EHD in the point-plane configuration in air at atmospheric pressure and room temperature and predicted the existence of an additional small vortex in the ionization layer by calculation [19]. Zheng et al. studied the electrical characteristics of a wire-plate electrostatic precipitator and the particle transport behavior with consideration of EHD and particle space charge by numerical simulation and the results indicate that the ionic wind in the electrostatic field weakened in the condition that the movement of ions is restricted for corona suppression [20]. Feng et al. utilized a hybrid method comprising of the Finite Element Method (FEM) for the simulation of the ionized electric field and the Finite Volume Method (FVM) for the prediction of the EHD flow in ESP simulation and analyzed quantitatively the corona induced EHD flow analyzed by the Qkubo-Weiss index [21].

In summary, the research on EHD at home and abroad mainly focuses on the coupling analysis of particle transport and flow field. This means that the influence of EHD on collection efficiency of ESP is obtained indirectly by the analysis on flow field distribution. Few studies have focused on the deposition pattern induced by EHD, which can provide valuable data for the optimal design of the structure and size of ESP, especially for the shape of the collection plate. In our work, a comprehensive multifield coupling model of wire-plate ESP was established by self-defined programming. The effects of EHD on collection efficiency of ESP and particle deposition pattern were studied, and the EHD behavior in a special needle-plate arrangement was analyzed. Relevant research results can provide reliable theoretical reference for the design and modification of large ESP in industry.

\section{Mathematical Model}

The gas flow, electric field, and particle transport inside ESP exert an influence on each other [22-25]. The interaction between them is shown in Figure 1. The EHD induced by electric field affects the particle transport by changing flow field first.

2.1. Electric Field. The electric field intensity in the wire-plate ESP can be described by Poisson equation [26].

$$
\begin{aligned}
& \nabla^{2} V=-\frac{\rho_{c}}{\varepsilon_{0}} \\
& -\nabla V=E
\end{aligned}
$$

where $V$ is potential; $\rho_{c}$ is space charge density; $\varepsilon_{0}$ is vacuum dielectric constant; $E$ is electric field intensity.

The charge density can be expressed as current continuity equation.

$$
\begin{aligned}
\nabla \bullet(J) & =0 \\
J & =\rho_{c}(b E+u)-D \nabla \rho_{c}
\end{aligned}
$$

where $J$ is current density; $b$ is ionic mobility; $u$ is gas velocity; $D$ is ionic diffusion coefficient. 


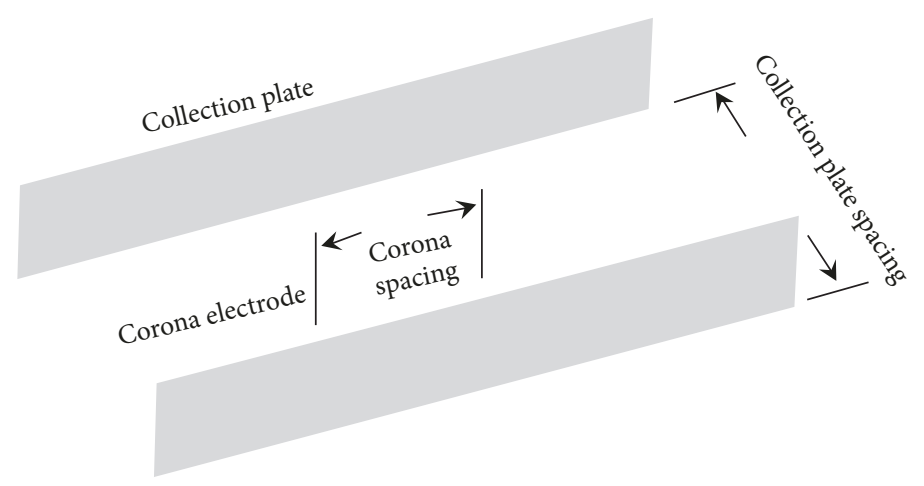

FIgURE 2: Three-dimensional geometric model of ESP.

2.2. Particle Transport. Momentum conservation equation of particles is as follows:

$$
\begin{aligned}
& \frac{d x_{p}}{d t}=u_{p} \\
& \frac{d u_{p}}{d t}=\frac{3 \rho C_{D}\left(u-u_{p}\right)\left|u-u_{p}\right|}{4 \rho_{p} d_{p}}+F_{E P}+m_{p} \mathrm{~g}
\end{aligned}
$$

where $x_{p}$ is the spatial position of particles; $u_{p}$ is the particle velocity; $\rho$ is the particle density; $C_{D}$ is the drag coefficient; $F_{E P}$ is electric field force acting on particles and can be expressed as $q_{p} E ; m_{p}$ is the mass of single particle.

2.3. Gas Flow. Mass conservation equation of gas phase is as follows:

$$
\frac{\partial}{\partial x_{k}}\left(\rho_{g} u_{k}\right)=0
$$

where $u_{k}$ is gas velocity; $\rho_{g}$ is gas density.

In view of the fact that the average random range of ion motion is small relative to the characteristic scale of ESP, the influence of EHD on flow field is assumed to be instantaneous and can be embodied by adding a volume force term of $\rho_{c} E$ in the gas phase momentum equation $[27,28]$.

Momentum conservation equation of gas phase is as follows:

$$
\frac{\partial}{\partial x_{k}}\left[\rho_{g} u_{i} u_{k}-\left(\mu+\mu_{t}\right) \frac{\partial u_{i}}{\partial x_{k}}\right]=-\frac{\partial P}{\partial x_{i}}+F_{D i}+\rho_{c} E
$$

where $\mu$ is the dynamic coefficient of viscosity; $\mu_{t}$ is the turbulent viscosity; $F_{D i}$ is the resistance force due to the relative motion between the gas and particles.

\section{Geometric Structure}

The geometric model of $3 \mathrm{D}$ ESP is shown in Figure 2, where the corona spacing is $0.24 \mathrm{~m}$ and the collection plate spacing is $0.4 \mathrm{~m}$. Particles are injected randomly without repetition from the inlet and are of no slip with gas. Considering the strong interaction between electric

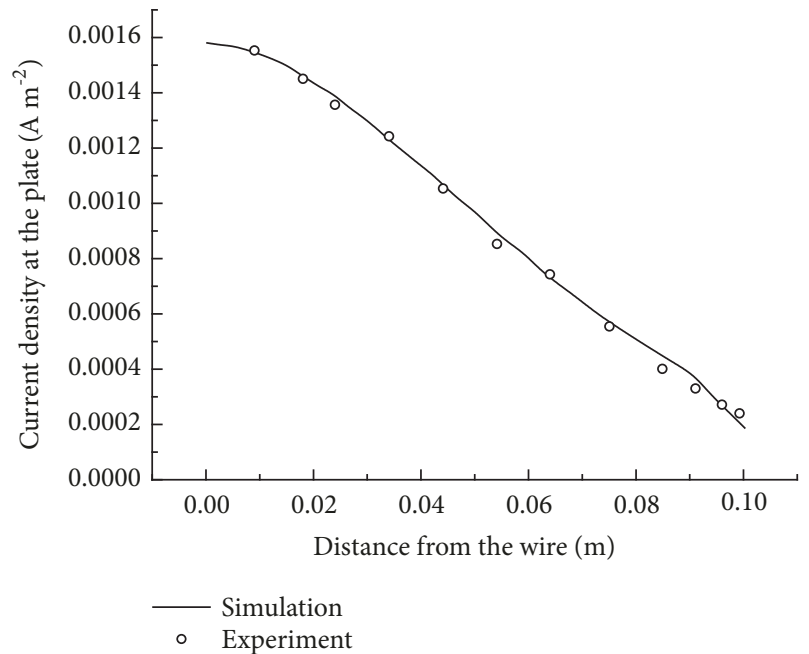

FIgURE 3: Comparison of simulation results with experiment data.

field and particle transport and the randomness of particle phase, the coupling between them is carried out by a small underrelaxation factor. The 2D-ESP model adopted in this work includes four corona electrodes to exhibit the ionic wind effect evidently; a detailed illustration is omitted here.

\section{Model Verification}

To validate the corona model which is related to the EHD characteristics, the simulation results of electric field without gas flow effect in 2D case are compared with the experimental data of wire-plate ESP [29], as shown in Figure 3. It can be seen from the graph that the simulated current density on the plate is in good agreement with experimental data, indicating that the corona model adopted in our work is in line with reality.

\section{Results and Discussion}

5.1. EHD Effect on Collection Efficiency. EHD primarily affects the gas flow, and the particle transport is influenced through gas-solid coupling. The residence time of particles 


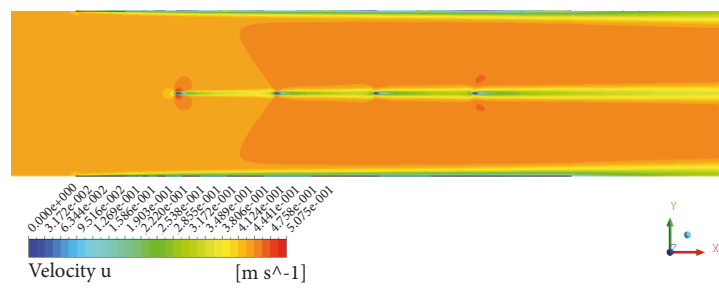

(a) No EHD

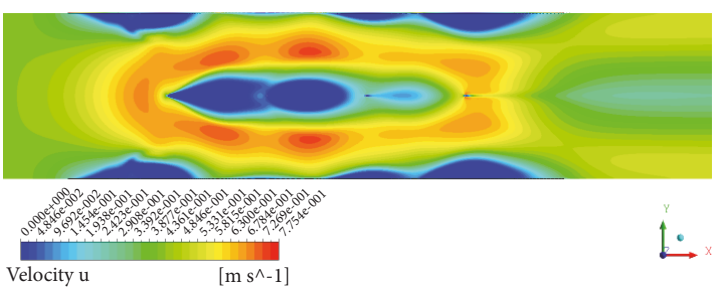

(b) With EHD

FIGURE 4: Profile of streamwise velocity of airflow.

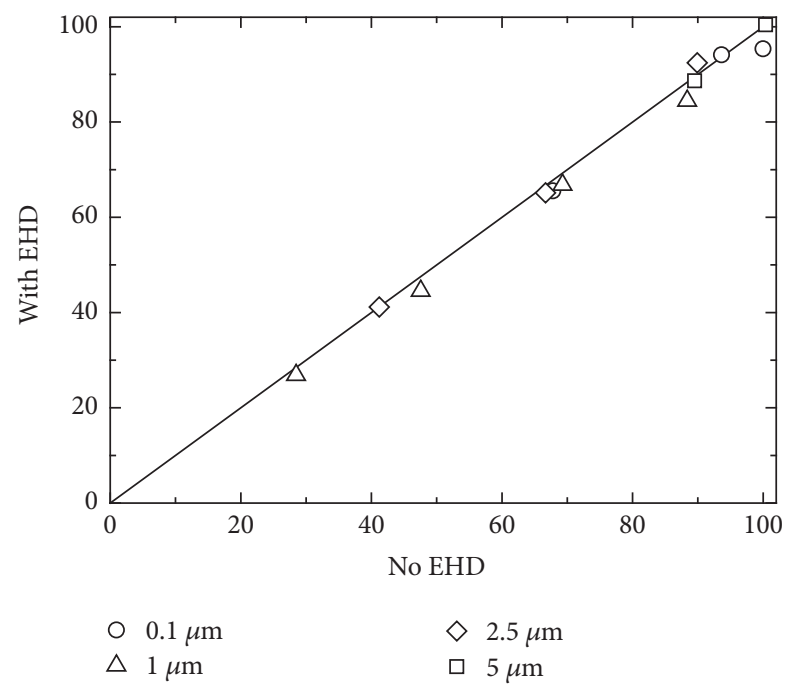

FIGURE 5: Collection efficiency of particles with different diameters.

in ESP depends on the streamwise velocity, which has a direct impact on collection efficiency of ESP. Figure 4 shows the streamwise velocity of airflow with or without EHD in 2D case where the voltage and velocity are fixed. As seen in Figure 4, the airflow velocity near the wall is small and keeps constant with no EHD effect, which contributes to the particle deposition on the collection plate. When considering the EHD effect, the airflow velocity near the wall becomes unstable, and velocity in local space becomes very large. This is because the electric force effect of EHD drives the gas moving from the corona wire towards the collection plate and makes the gas circulate in local space of ESP, resulting in a decrease of the airflow area, and thus the airflow velocity near the collection plate increases.

The collection efficiency of particles with different diameters in the presence or absence of EHD is shown in Figure 5. It can be obtained from the graph that the collection efficiency with EHD effect is lower than that without EHD effect; this is because the airflow circulation caused by EHD increases the airflow velocity near the wall and causes the particles to escape faster from ESP, which is not conducive to the particle deposition; the negative effect of EHD on collection efficiency of small particles is greater than that of big particles. This is due to the fact that the EHD affects particle transport through gas-solid coupling, and the small particles have small inertia and good airflow following, which makes them more sensitive to airflow variation.

5.2. EHD Effect on Particle Deposition. The particle deposition pattern has three-dimensional characteristics. Therefore, the 3D-ESP structure is built to characterize the particle deposition process with or without EHD.

The trajectory of particles with uniform diameter of $2.5 \mu \mathrm{m}$ in the presence or absence of EHD in 3D-ESP is shown in Figure 6, colored by the particle charge. It can be concluded from the figure that when EHD is not available, the particle trajectory is basically straight, and certain regularity shows up. When EHD is considered, the particle motion becomes irregular and the turbulence intensity increases.

Figures 7 and 8 are the particle deposition rate when $\mathrm{v}=0.25 \mathrm{~m} / \mathrm{s}$ or $0.5 \mathrm{~m} / \mathrm{s}$, respectively. It can be found from the figures that EHD has a significant effect on particle deposition no matter what the flow velocity is. And the effect of EHD on particle deposition rate is greater at low airflow velocity.

5.3. EHD Effect in the Case of Needle Pointing Plate. When the needle tip points to the plate, the trajectory of particles with uniform diameter of $2.5 \mu \mathrm{m}$ is shown in Figure 9, colored by particle charge. Because the needle tip is oriented towards the collection plate, the ions produced by corona discharge cannot reach the tip root, i.e., the symmetrical axis in streamwise direction of ESP. Therefore, a region without free ions appears near the symmetrical axis. From Figure 9, one can obtain that, when EHD is switched off, the charge of particles passing through the nonionic region is very small, which indicates that particles cannot obtain enough charge in the region. When EHD effect is considered, the particle trajectory changes significantly and the particles charge more when passing near the corona wire. This is because EHD makes the airflow circulate locally near the corona wire and reduces the number of particles passing through the nonionic region. Due to the airflow circulation, some particles move from the nonionic region to the collection plate and are then charged.

Figure 10 shows the collection efficiency of $2.5 \mu \mathrm{m}$ particles with or without EHD effect when the needle points to the plate. As can be seen from the figure, the collection efficiency with EHD is slightly higher than that without EHD. This is because the existence of EHD reduces the number of particles passing through the nonionic region and increases the number of fully charged particles in the case of 


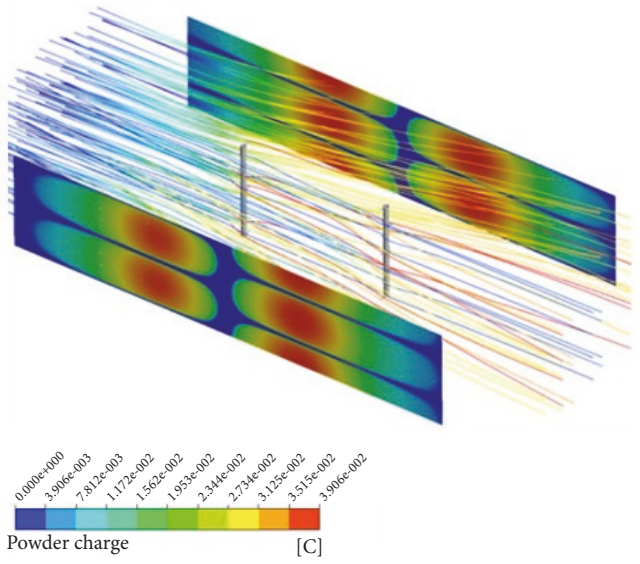

(a) No EHD

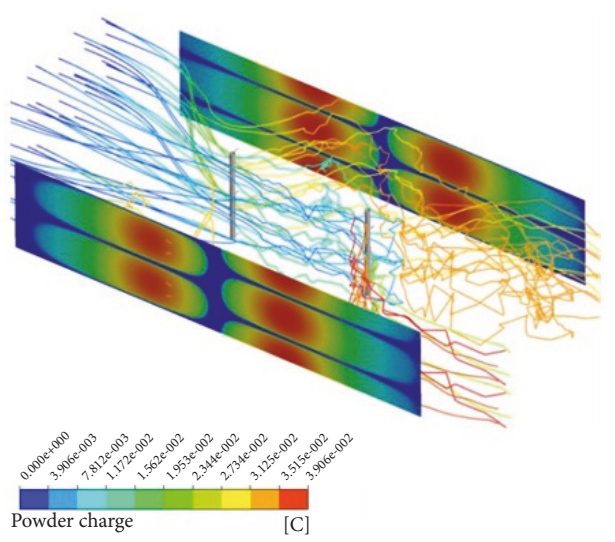

(b) With EHD

FiguRE 6: Trajectory of particles with uniform diameter of $2.5 \mu \mathrm{m}$.
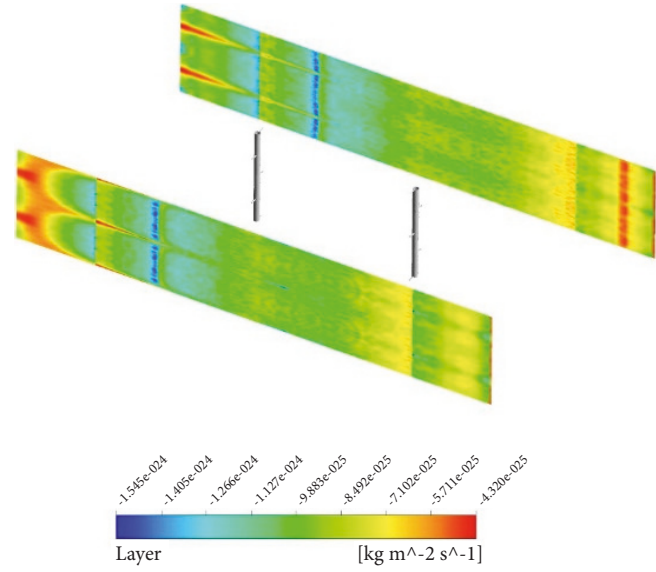

(a) No EHD
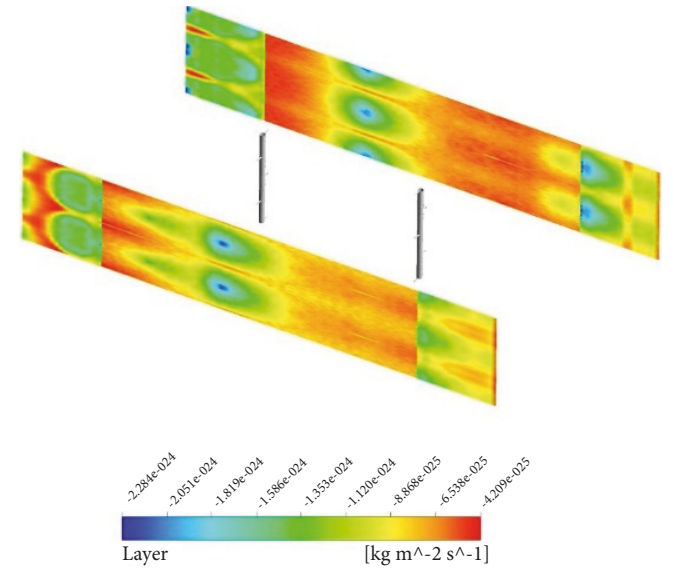

(b) With EHD

Figure 7: Deposition rate of particles at $v=0.25 \mathrm{~m} / \mathrm{s}$.

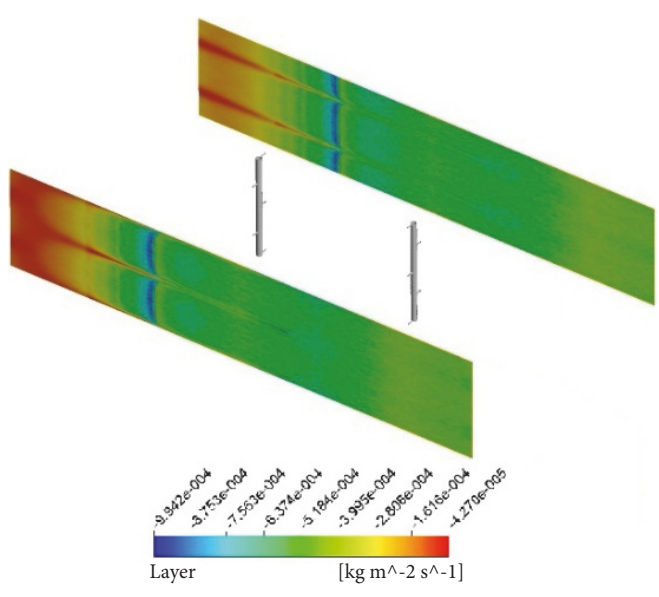

(a) No EHD

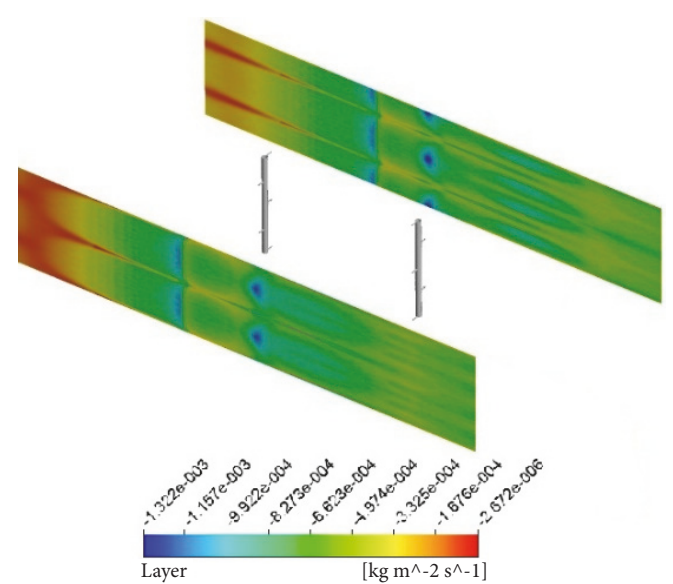

(b) With EHD

Figure 8: Deposition rate of particles at $v=0.5 \mathrm{~m} / \mathrm{s}$. 


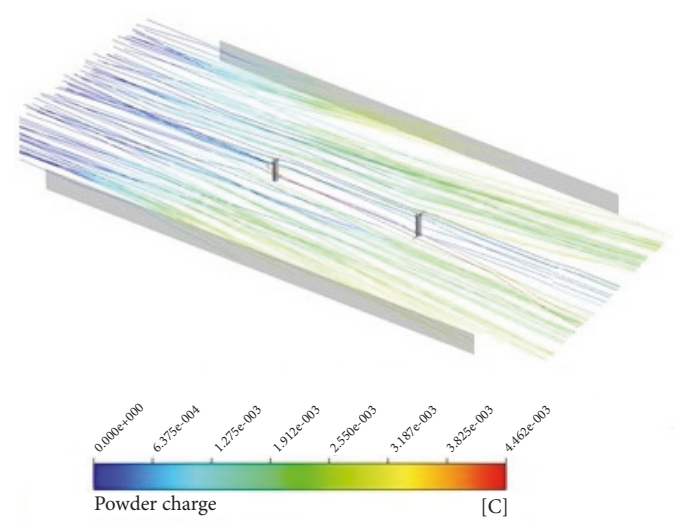

(a) No EHD

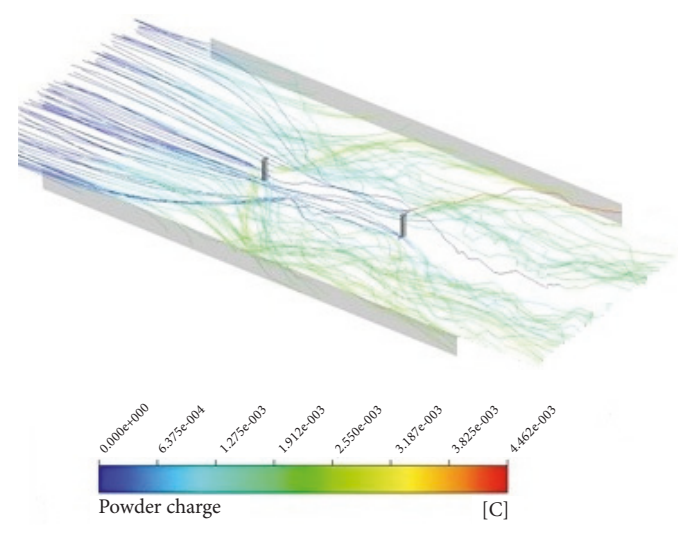

(b) With EHD

FIGURE 9: Trajectory of particles in the case of needle pointing plate.

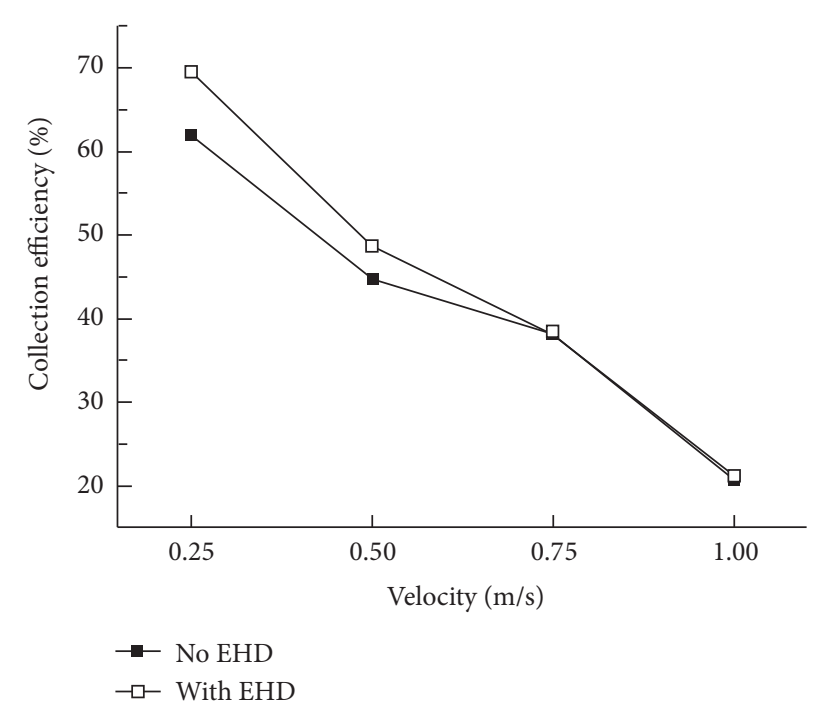

Figure 10: Collection efficiency of particles at different velocities in the case of needle pointing plate.

needle pointing to the plate. Under the influence of electric field, fully charged particles move towards the plate and are trapped by collection plate, thus improving the collection efficiency. And with the decrease of flue gas velocity, the enhancement of collection efficiency due to ionic wind is greater.

\section{Conclusions}

On the basis of self-defined programming, a comprehensive multifield coupling model of ESP was established, and the influence of EHD on particle transport and deposition pattern was analyzed. The conclusions are as follows: Generally, when the EHD is switched on, the airflow area of the airflow decreases, the streamwise velocity of airflow near the collection plate obviously increases, and the residence time of particles in ESP is shortened, which results in a decline of the collection efficiency of ESP; the effect of EHD on particle deposition pattern cannot be ignored and is more obvious at lower flue gas velocity; in the case of needle tip pointing to the collection plate, the EHD contributes to the improvement of collection efficiency of ESP by the transportation of particles in nonionic region to the ionic region.

\section{Data Availability}

The data used to support the findings of this study are available from the corresponding author upon request.

\section{Conflicts of Interest}

The authors declare that they have no conflicts of interest.

\section{Acknowledgments}

The authors are grateful to the National Key Research and Development Program of China (2016YFC0203703) for the financial support of current work.

\section{References}

[1] M. J. Quina, J. C. Bordado, and R. M. Quinta-Ferreira, "Treatment and use of air pollution control residues from MSW incineration: an overview," Waste Management, vol. 28, no. 11, pp. 2097-2121, 2008.

[2] H. Bin, Y. Yang, L. Cai, Y. Zhulin, S. Roszak, and Y. Linjun, "Experimental study on particles agglomeration by chemical and turbulent agglomeration before electrostatic precipitators," Powder Technology, vol. 335, pp. 186-194, 2018.

[3] E. Jaroudi, I. Sretenovic, G. Evans, and H. Tran, "Factors affecting particulate removal efficiency of kraft recovery boiler electrostatic precipitators: a technical review," TAPPI Journal, vol. 17, no. 05, pp. 273-283, 2018.

[4] A. Jaworek, A. Sobczyk, A. Krupa, A. Marchewicz, T. Czech, and L. Śliwiński, "Hybrid electrostatic filtration systems for fly ash particles emission control. A review," Separation and Purification Technology, vol. 213, pp. 283-302, 2019.

[5] P. Magnier, D. Hong, A. Leroy-Chesneau, J. Bauchire, and J. Hureau, "Control of separated flows with the ionic wind 
generated by a DC corona discharge," Experiments in Fluids, vol. 42, no. 5, pp. 815-825, 2007.

[6] R. Ohyama, K. Aoyagi, Y. Kitahara, and Y. Ohkubo, "Visualization of the local ionic wind profile in a DC corona discharge field by laser-induced phosphorescence emission," Journal of Visualization, vol. 10, no. 1, pp. 75-82, 2007.

[7] J. M. Johnson and D. B. Go, "Recent advances in electrohydrodynamic pumps operated by ionic winds: a review," Plasma Sources Science and Technology, vol. 26, no. 10, Article ID 103002, 2017.

[8] H. Shen, W. Yu, H. Jia, and Y. Kang, "Electrohydrodynamic flows in electrostatic precipitator of five shaped collecting electrodes," Journal of Electrostatics, vol. 95, pp. 61-70, 2018.

[9] J. Podliński, M. Danowska, T. Izdebski, and M. Dors, "Electrohydrodynamic pump supplied by unipolar direct current voltage with both positive and negative corona discharge," Journal of Fluids Engineering, vol. 141, no. 1, Article ID 011206, 2019.

[10] Y. M. Kang, M. L. Rong, and H. G. Shen, "Corona discharge and ionic wind in air cleaning processes," Nature Magazine, vol. 1, pp. 63-71, 2002.

[11] T. Yamamoto, S. Maeda, Y. Ehara, and H. Kawakami, "Development of EHD-assisted plasma electrostatic precipitator," IEEE Transactions on Industry Applications, vol. 49, no. 2, pp. 672678, 2013.

[12] N. Zehtabiyan-Rezaie, M. Saffar-Avval, and K. Adamiak, "Numerical investigation of water surface deformation due to corona discharge," Journal of Electrostatics, vol. 96, pp. 151-159, 2018.

[13] B. C. Choi and P. J. Park, "A 1D ionic transport model for nonlinear response analysis of a counterflow laminar diffusion flame in DC electric fields," Fuel, vol. 233, pp. 610-619, 2018.

[14] G. L. Leonard, M. Mitchner, and S. A. Self, "An experimental study of the electrohydrodynamic flow in electrostatic precipitators," Journal of Fluid Mechanics, vol. 127, no. -1, p. 123, 1983.

[15] J. Davidson and P. McKinney, "EHD flow visualization in the wire-plate and barbed plate electrostatic precipitator," IEEE Transactions on Industry Applications, vol. 27, no. 1, pp. 154-160.

[16] G. A. Kallio and D. E. Stock, "Interaction of electrostatic and fluid dynamic fields in wire-plate electrostatic precipitators," Journal of Fluid Mechanics, vol. 240, pp. 133-166, 1992.

[17] S. Bernstein and C. T. Crowe, "Interaction between electrostatics and fluid dynamics in electrostatic precipitators," Environment International, vol. 6, no. 1-6, pp. 181-189, 1981.

[18] L. Zhao and K. Adamiak, "Numerical Simulation of the Electrohydrodynamic Flow in a Single Wire-Plate Electrostatic Precipitator," IEEE Transactions on Industry Applications, vol. 44, no. 3, pp. 683-691, 2008.

[19] S. Mantach and K. Adamiak, "A double-vortex EHD flow pattern generated by negative corona discharge in point-plane geometry," Journal of Electrostatics, vol. 93, pp. 118-124, 2018.

[20] C. Zheng, X. Zhang, Z. Yang et al., "Numerical simulation of corona discharge and particle transport behavior with the particle space charge effect," Journal of Aerosol Science, vol. 118, pp. 22-33, 2018.

[21] Z. Feng, Z. Long, S. Cao, and K. Adamiak, "Characterization of electrohydrodynamic (EHD) flow in electrostatic precitators (ESP) by numerical simulation and quantitative vortex analysis," Journal of Electrostatics, vol. 91, pp. 70-80, 2018.

[22] N. Farnoosh, K. Adamiak, and G. Castle, "Numerical calculations of submicron particle removal in a spike-plate electrostatic precipitator," IEEE Transactions on Dielectrics and Electrical Insulation, vol. 18, no. 5, pp. 1439-1452, 2011.

[23] K. Luo, Y. Li, C. Zheng, X. Gao, and J. Fan, "Numerical simulation of temperature effect on particles behavior via electrostatic precipitators," Applied Thermal Engineering, vol. 88, pp. 127-139, 2015.

[24] M. Dong, F. Zhou, Y. Zhang, Y. Shang, and S. Li, "Numerical study on fine-particle charging and transport behaviour in electrostatic precipitators," Powder Technology, vol.330, pp. 210 218, 2018.

[25] H. Kim, M. Kim, B. Han et al., "Fine particle removal by a twostage electrostatic precipitator with multiple ion-injection-type prechargers," Journal of Aerosol Science, vol. 130, pp. 61-75, 2019.

[26] H. Schmid and L. Vogel, "On the modelling of the particle dynamics in electro-hydrodynamic flow-fields: I. Comparison of Eulerian and Lagrangian modelling approach," Powder Technology, vol. 135-136, pp. 118-135, 2003.

[27] A. Soldati, "on the effects of electrohydrodynamic flows and turbulence on aerosol transport and collection in wire-plate electrostatic precipitators," Journal of Aerosol Science, vol. 31, no. 3, pp. 293-305, 2000.

[28] N. Farnoosh, K. Adamiak, and G. Castle, "3-D numerical analysis of EHD turbulent flow and mono-disperse charged particle transport and collection in a wire-plate ESP," Journal of Electrostatics, vol. 68, no. 6, pp. 513-522, 2010.

[29] S. Abdel-Satar and H. Singer, "Electrical conditions in wire-duct electrostatic precipitators," Journal of Electrostatics, vol. 26, no. 1, pp. 1-20, 1991. 


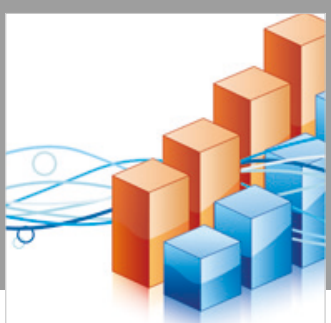

Advances in

Operations Research

\section{-n-m}
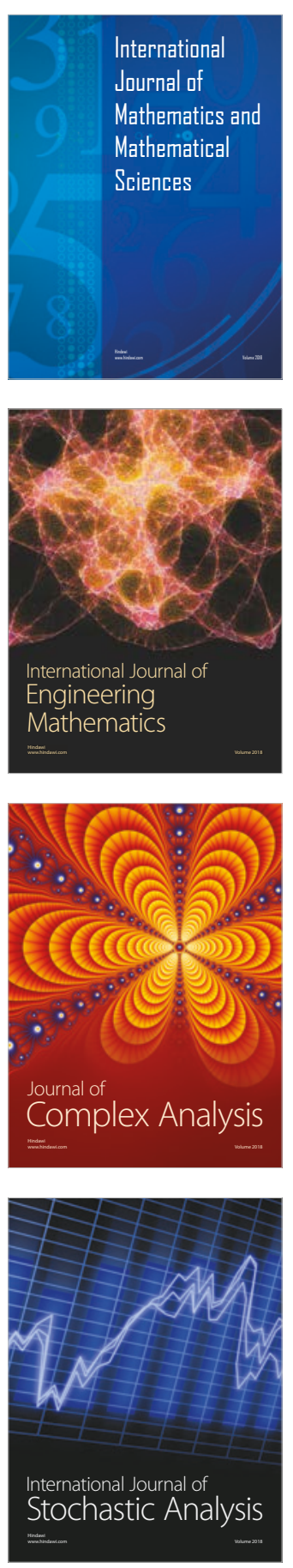
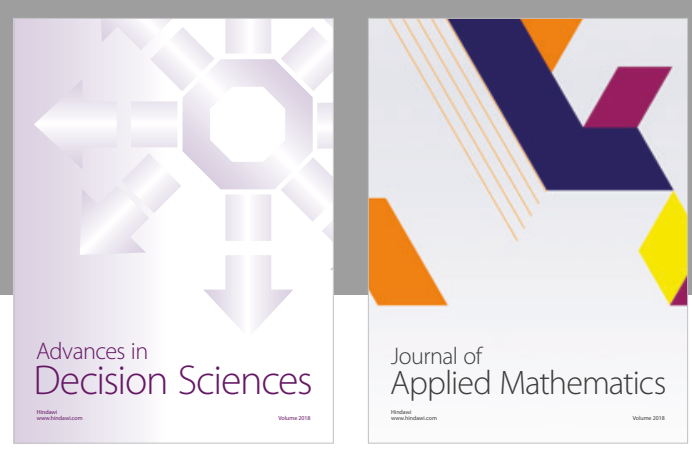

Journal of

Applied Mathematics
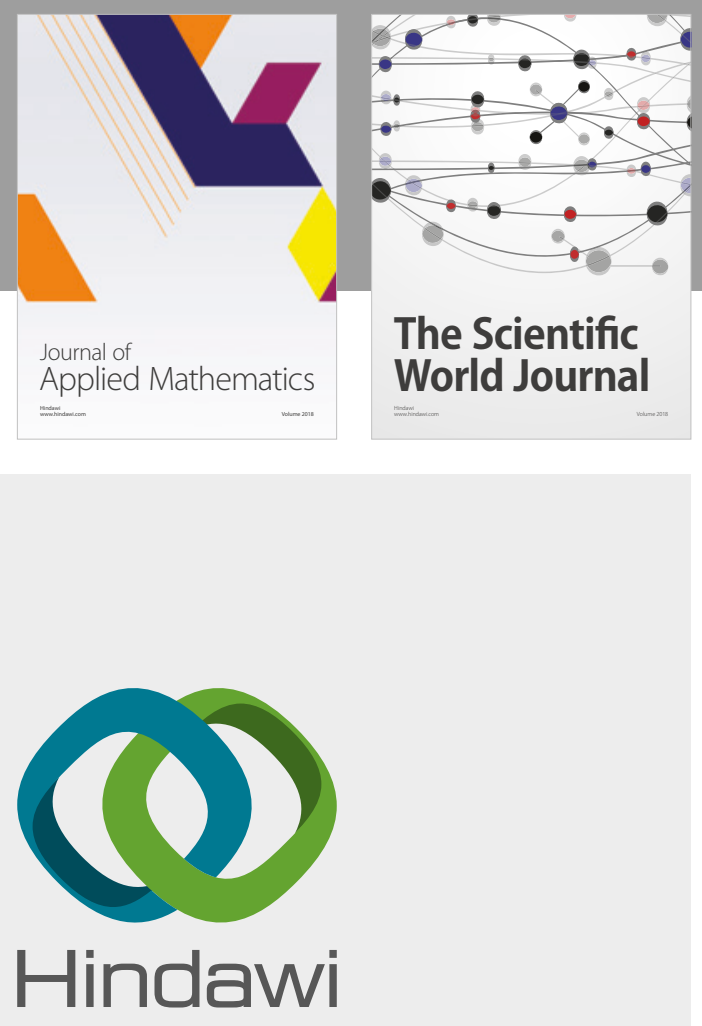

Submit your manuscripts at

www.hindawi.com

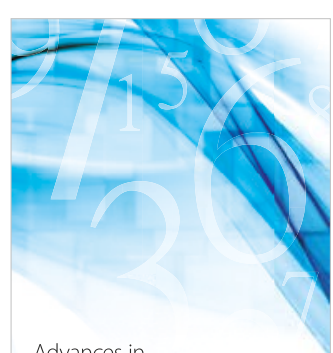

Advances in
Numerical Analysis
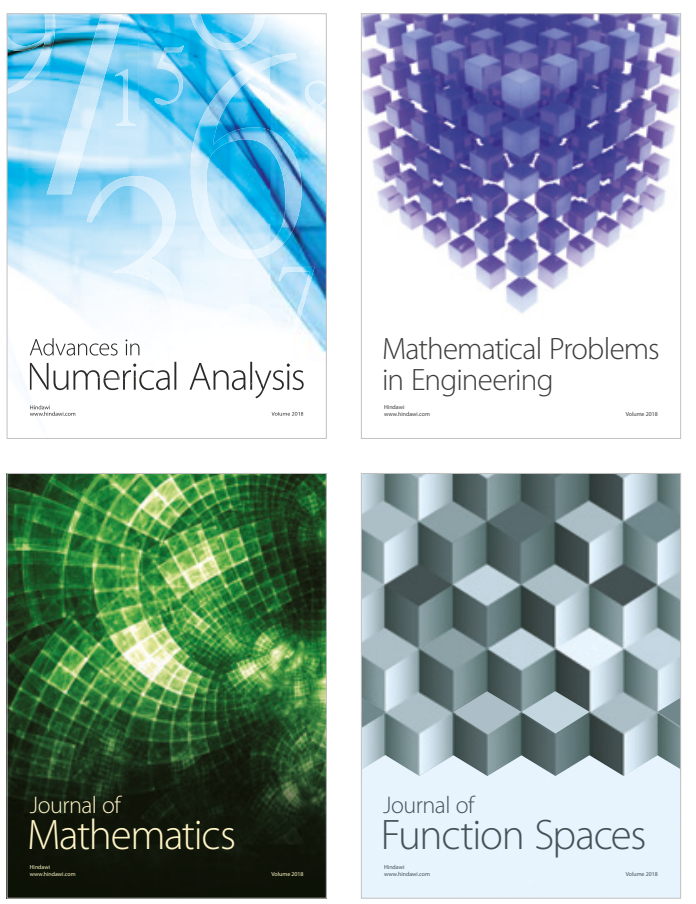

Mathematical Problems in Engineering

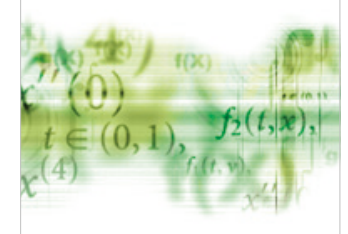

International Journal of

Differential Equations

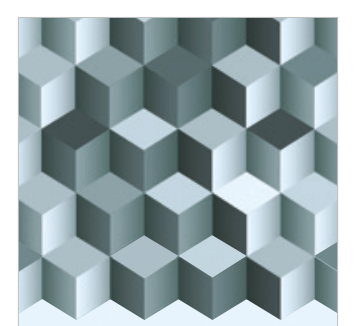

Journal of

Function Spaces

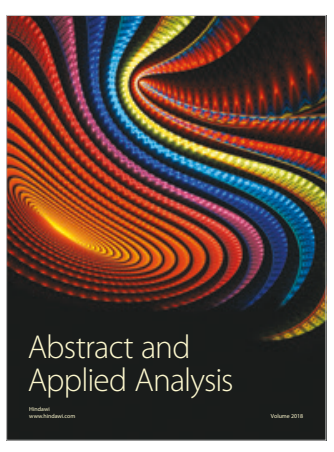

The Scientific

World Journal

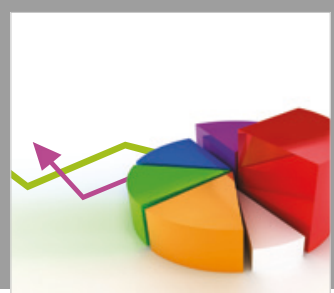

Journal of

Probability and Statistics
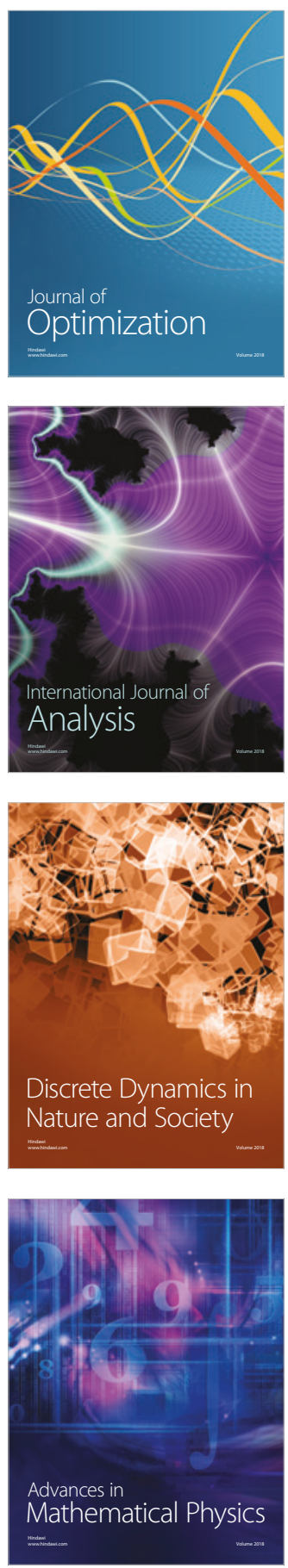